\title{
CONEXIÓN ENTRE LA ESCUELA Y OTRAS INSTITUCIONES DE LA COMUNIDAD
}

Ana Sofia Vargas Hernández ${ }^{1}$

\section{Resumen}

La escuela es una de las muchas instituciones que pertenecen al Estado, por lo tanto, no se puede pensar que está aislada, aunque algunos piensen que sí. Las relaciones que se establecen entre la escuela y otras instituciones de la comunidad enriquecen el proceso educativo, lo hacen más dinámico y más agradable. Este artículo señala cómo se pueden aprovechar los vínculos existentes entre las distintas instituciones de una sociedad con la escuela, si se considera a esta como la encargada de preparar a la niñez $y$ a la juventud para que puedan enfrentar y desarrollar, exitosamente, su papel en los entomos social, educativo, político, económico y religioso.

Palabras claves: escuela, comunidad, instituciones estatales.

\section{Introducción}

esde el momento en que se creó la institución educativa, se tuvo la idea de que sería para el beneficio de los sujetos participantes, esto es: estudiantes, docentes, padres de familia y comunidad. De alguna manera, ha sido así, pues todos obtienen alguna cuota de ganancia al concluir la formación de los

1 Profesora de Preecolar bilingüe en Montessori Nursery and Preschool, Santa Ana, Costa Rica. 
educandos. Estos son, en todo sentido, los primeros que van a beneficiarse con el proceso educativo y si ellos ganan, por supuesto que sus profesores y padres también lo harán; si ellos se forman apropiadamente, su comunidad se verá favorecida con su desempeño futuro como ciudadanos de su país y promotores del cambio, tanto en el ámbito nacional como en el internacional.

Por lo tanto, puede afirmarse que entre la escuela, los participantes y otras instituciones existe una relación indisoluble. Los planes, las decisiones y las consecuencias de una parte afectan e influyen en las otras; así pues, es imprescindible que lo que se lleve a cabo y lo que se tome en cuenta para establecer esa relación, se haga de la mejor manera y, así, será positivo para todos.

Este artículo tiene como objetivo dar cuenta de la importancia de conocer las relaciones que existen entre la escuela y algunas instituciones ${ }^{2}$, tanto en el entorno regional, el cual puede ser distrital o cantonal, como provincial, nacional y hasta internacional. Asimismo, se persigue conocer cuáles son los entes que se vinculan, constantemente, con la institución escolar y cuáles lo hacen esporádicamente.

La razón por la cual se ha realizado la investigación de este tema y se presenta este documento, es porque, por lo general, los individuos, aunque estén en contacto con la educación, no perciben la conexión que se da entre la institución educativa y otras, sean públicas, privadas o gubernamentales. La mayoría de las personas cree que la escuela sólo se enlaza con el Ministerio de Educación Pública (MEP).

Este escrito se realizó con base en la información obtenida de la poca bibliografía existente al respecto y con algunos datos extraídos del, también muy escaso, material recopilado en Internet. Para complementar estos pocos datos se aplicó una encuesta (véase anexo) a diez directores: cinco de escuelas públicas y cinco de escuelas privadas, para poder realizar afirmaciones con opiniones reales.

\section{Generalidades sobre el nexo entre la escuela y otras instituciones}

Para hablar de la relación existente entre la escuela y la comunidad ${ }^{3}$ hay que pensar un poco acerca del origen de cada una de ellas. Las comunidades

\footnotetext{
2 Se debe aclarar que serán algunas instituciones, pues es muy dificil poder abarcar absolutamente todas aquellas que se relacionan, que influyen y que son influidas por la relación con la institución escolar.

3 Entiéndase comunidad como el conjunto de instancias, instituciones y grupos de cualquier tipo existentes en ella. El ámbito de la comunidad no se puede definir claramente, ya que puede variar desde un sector geográfico muy pequeño hasta un alcance intemacional.
} 
fueron apareciendo conforme los individuos sintieron la necesidad de agruparse, para protegerse y para compartir todos aquellos productos y aspectos que se producían y se daban dentro de ese grupo organizado. Comunidad puede tomarse como sinónimo de grupo social; aunque se habla, más a menudo, de sociedad, que de grupo social o de comunidad.

La escuela, como tal, nació cuando las sociedades vieron la necesidad de transmitir los conocimientos generales y la cultura a los niños y jóvenes, con el fin de que se formaran y se integraran, con propiedad, al grupo en el que nacieron.

\section{Escuela: institución de la comunidad}

Si bien se distingan la escuela, por un lado, y la comunidad, por otro, es necesario aclarar que se hace por asunto de facilidad para entender la relación entre ellas. Pero, es lógico, que si la escuela está inmersa en un grupo es parte de él, es una de las instituciones que en él coexisten. Al respecto, Cárdenas (1990) afirma que la escuela de la comunidad ve a la sociedad como un todo, del que la escuela es una parte importante; además, que la actividad de la escuela debe responder a los intereses y a las necesidades de la comunidad y, también, que la escuela debe identificarse con la cultura y con la acción del grupo en el que se encuentra.

Desde que un individuo nace en un grupo determinado, comienza a empaparse de ciertos conocimientos y costumbres que le van dando una personalidad específica y lo van formando para el futuro desempeño como ciudadano; es decir, va adquiriendo la cultura propia de la comunidad, para poder desarrollarse apropiadamente en ella y establecer una adecuada relación, con todas las personas con las que comparte.

\section{Función de la escuela}

La sociedad influye en las personas que en ella viven, para que adopten determinado comportamiento, ciertos conocimientos y una cultura específica, lo que hace que tengan una actuación especial y diferente de otros grupos. Esa forma de ser de los individuos es con la que se enfrentan los docentes en las aulas; por eso, se puede afirmar que la sociedad influye en la escuela, a veces positiva, a veces negativamente.

La función de la escuela va más allá de sus muros y llega a las otras instituciones de la comunidad, a las que influye de distintas maneras y se puede 
advertir que muchos de los cambios que ocurren en la comunidad surgen de la escuela, pues entre estas se da la más completa integración e interacción (Cárdenas, 1990); sin embargo, como dice Cassany (1999), entre la escuela y la comunidad se da una relación, no una fusión, pues la escuela maneja una clara definición, un mantenimiento de roles y una serie de criterios institucionales; y la comunidad tiene objetivos más generales, ya que debe abarcar los objetivos, las funciones y los intereses de todas las instituciones que en ella existen. Ambas se influyen mutuamente, pero una no sustituye nunca a la otra.

\section{Ámbito de influencia de la escuela}

El ámbito de influencia de la institución educativa, generalmente, depende del nivel académico; así, el espacio afectado por la educación primaria es muy limitado, pues abarca un sector geográfico cercano a la escuela, esto es lo distrital; las instituciones de enseñanza secundaria comprenden un ámbito más amplio, su influencia llega a un espacio cantonal y, a veces, regional. Por último, la universidad expande aún más su acción y abarca a la comunidad provincial y, muy a menudo, nacional (Cárdenas, 1990).

En algunas ocasiones, la escuela tiene que luchar contra la comunidad, en el sentido en que debe corregir, cambiar o desechar muchos hábitos, ideas e influencias negativas que los educandos han adquirido a través de los años en los ambientes en que se han desenvuelto.

\section{Modalidades educativas}

En Costa Rica, el sistema escolar se encuentra dividido en tres modalidades, a saber: la educación oficial o pública, la educación privada y la semiprivada ${ }^{4}$. En este trabajo se tomó en cuenta la opinión de los directores de algunas escuelas privadas y públicas, con el fin de tener el criterio de las dos modalidades más numerosas existentes en el país, que son las que mencionan García Hoz y Medina Rubio (1987) cuando aclaran que existe el sistema escolar oficial y, en los países donde se da una mínima libertad de enseñanza, existen también sistemas escolares privados. Como estos autores no tocan la modalidad semiprivada y en Costa Rica es minoritaria, se prefirió no trabajar con las opiniones emanadas de docentes que laboran en ella.

4 Se llama educación semiprivada a la que se ofrece en instituciones escolares fundadas por una persona o un grupo de ellas de manera privada, y que luego dejó esta modalidad y se ofreció la infraestructura al MEP, con la condición de que éste pagara los salarios del personal que ahí labora. 
En los siguientes apartados, se verán, en forma separada, las relaciones de la comunidad con la escuela pública y con la escuela privada. Aunque las relaciones son muy parecidas, sí se dan algunas diferencias, pues los objetivos son diferentes.

\section{Relaciones entre escuela pública y otras instituciones}

Según García Hoz y Medina Rubio (1987), no se debe creer que la educación se lleva a cabo sólo en los centros docentes, pues esta es una tarea viva que actúa todo el tiempo y se produce en todas las situaciones de la vida. Por esta razón, es tan importante que la buena educación comience desde el hogar, de manera informal y que, en el futuro, se complemente con la educación formal, así como con los conocimientos y las experiencias que puedan obtenerse, mediante la relación de la institución educativa y otras instituciones de la comunidad.

Es necesario que se lleven a cabo esas relaciones, pues, en su origen, la escuela nació sólo con el fin de transmitir conocimientos, de enseñar cosas nuevas; en el presente, se sabe que uno de los fines de la educación es preparar a los y las estudiantes, para que puedan insertarse, social y productivamente, a la sociedad humana. La escuela es casi, como lo afirman García Hoz y Medina Rubio (1987), una situación de tránsito, como un puente entre la familia y la sociedad en general.

A pesar de que en este documento no se va hacer la división correspondiente, sí hay que aclarar que las instituciones con las que puede relacionarse la escuela pueden ser de dos tipos: gubernamentales y no gubernamentales; estas últimas se dividen en dos grupos: las públicas y las privadas.

La escuela pública tiene algunas limitaciones, por lo general, económicas, para relacionarse con otras entidades; sin embargo, trata de llevar a cabo una serie de conexiones para posibilitar a sus educandos la inserción en la realidad, lo que les ayudará a desenvolverse mejor con sus semejantes dentro del grupo social al que pertenecen y con otros grupos que les puedan brindar una forma de ganarse la vida en el futuro.

\section{Relación escuela-MEP}

Según todos los directores encuestados, la relación obligatoria.y necesaria que tiene la escuela es con el MEP, pues depende de él para su funcionamiento; es el que les provee los recursos humanos y económicos 
para cumplir con los objetivos propuestos. Aunque, como dicen algunos, no logran de él, todo lo que necesitan, por lo menos se dan el gusto de pedir y se hacen la ilusión de que van a obtener lo solicitado.

Esta relación con el MEP es administrativa en el nivel de los funcionarios y, totalmente, académica, en el caso de los y las estudiantes, ya que es el que impone las normas de organización, de disciplina, de planeamiento y de evaluación.

\section{Relación escuela-Municipalidad}

La escuela también tiene nexos con la Municipalidad. Esta relación es muy beneficiosa, pues no se limita sólo a ofrecer y a recibir los servicios que esa entidad tiene a su cargo y a cobrar lo que corresponde por ellos, sino que algunos municipios tienen un sistema de becas para estudiantes sobresalientes y de muy escasos recursos económicos. Estos pueden llenar la solicitud respectiva, si consideran que poseen los requisitos; posteriormente, en la oficina encargada de otorgar este beneficio realizan las entrevistas y los estudios de rigor para otorgarlo a los estudiantes que más lo necesiten y más lo merezcan. El aporte monetario no es muy alto, pero sí es significativo para una persona que desea superarse en el campo académico; eso los ayuda a seguir con sus estudios.

Otra forma de relación con esta entidad son las visitas que los estudiantes realizan a sus instalaciones cuando estudian, con sus docentes, los servidores de la comunidad.

\section{Relación escuela-Iglesia}

Entre la escuela y la Iglesia se mantiene una moderada, pero importante, relación, la cual tiene que ver, específicamente, con la orientación cristiana. Los curas, las y los catequistas, y los laicos comprometidos llegan, de vez en cuando, al centro educativo para ofrecer charlas a docentes y a estudiantes; para oficiar misa en alguna ocasión especial y, sobre todo, para motivar a que asistan al templo a confesarse, durante ciertas épocas del año, como son Semana Santa y final de año, para concluir el curso lectivo dando gracias a Dios.

\section{Relación escuela-Cuerpo de Bomberos}

Una oportunidad más que tiene la escuela de relacionarse con otros servidores públicos es cuando existe un Cuerpo de Bomberos en la comunidad, 
pues permite que grupos de escolares visiten sus instalaciones, con el fin de que conozcan cuál es su funcionamiento y, particularmente, para promover la seguridad en los diferentes ámbitos en los que se mueven los menores. Los orientan para evitar aquellos productos que podrían ser causantes de incendios, con el fin de que no se expongan a posibles quemaduras y no tener que lamentarse toda la vida.

Una de las actividades que se realizan en el Cuerpo de Bomberos es enseñarles con cuáles equipos combaten los incendios y cuál es la indumentaria que usan; esto con el fin de que los niños y las niñas conozcan lo que hace un bombero en una situación de incendio; pues algunos niños, en una conflagración, se han asustado al ver a un personaje con un traje tan extraño y con una máscara en la cara; quizá por eso no se acercan a ellos y se han internado en las llamas, que les han producido serios daños. Estas explicaciones han dado muy buen resultado.

\section{Relación escuela-museos}

Por otro lado, algunos temas de Estudios Sociales son recibidos y asimilados con más placer, si para ello se utilizan materiales reales; por esto se realizan visitas, con guía, al Museo Nacional y al Museo del Niño, entre otros. Los estudiantes se motivan mucho, participan y aprenden de manera efectiva, pues fijan mejor los conocimientos.

\section{Relación escuela-Ministerio de Salud}

Otra relación muy positiva y constante que establecen las escuelas es con el Ministerio de Salud, el cual se encarga de promover los buenos hábitos de salud, de alimentación y de higiene. Esta institución tiene un papel importante durante todo el año, pero, especialmente, en aquellas épocas cuando brotan algunas enfermedades, pues es la encargada de vacunar a la población, sobre todo, a aquellas personas (niños, niñas y personas de la tercera edad) que son más propensas a enfermarse.

\section{Relación escuela-Instituto Nacional de Seguros}

Por último, una institución que, de alguna manera, se conecta con la escuela por el objetivo de su relación, es el Instituto Nacional de Seguros (INS), el cual ofrece pólizas de seguros estudiantiles, tanto de enfermedad y 
accidente, como de vida. Estos planes protegen a los estudiantes durante las 24 horas y durante todo el curso lectivo.

\section{Relaciones entre escuela privada y otras instituciones}

No significa que con esta última se completan las instituciones que tienen alguna relación con la escuela, pues se podrían mencionar algunas más; sin embargo, para efectos de este trabajo y por la información recopilada con los directores, no se va a mencionar ninguna otra, por lo menos en lo que se refiere a la escuela pública.

$\mathrm{Al}$ igual que la escuela pública, la privada también mantiene relaciones con una gran cantidad de instituciones de la comunidad, la mayoría de las cuales coinciden con las que se unen a las instituciones estatales para llevar a cabo el proceso educativo. Sin embargo, el aprovechamiento de estos nexos en ambos sistemas es muy diferente, pues la pública tiene muchas necesidades y espera sacar ventaja de las otras instituciones en varios sentidos; en cambio, la privada, en algunos casos, debe relacionarse por obligación, porque así lo exige el sistema, no por necesidad.

\section{Relación escuela-MEP}

Esta unión obligada puede verse ilustrada en la relación que tiene la escuela privada con el MEP. Se sabe que este Ministerio es el encargado de dictar las normas de funcionamiento y hay que cumplirlas; pero no es la misma relación que tiene este ente con la escuela pública, pues existe en el Ministerio una oficina especializada, que se encarga de todos los asuntos y los lineamientos de la educación en las instituciones privadas. La relación es, exclusivamente, administrativa y académica.

\section{Relación escuela-Municipalidad}

La conexión existente con la Municipalidad también varía en esta modalidad de escuelas, pues se aprovecha lo que en sí representa esta institución para la comunidad y, por ende, para la escuela. Se aprecia y se estudia el servicio que brinda, así como la forma de organización que recuerda la primera que se dio en Costa Rica: el cabildo ${ }^{5}$, el cual todavía se da en algunos pueblos,

5 Ayuntamiento. Corporación que rige un municipio. 
cuando se debe tomar una decisión o se busca solucionar algún problema que afecta a todos.

\section{Relación escuela-Iglesia}

La Iglesia está presente en esta modalidad de escuelas, pues en muchas de las instituciones privadas existe una capilla o, por lo menos, un espacio muy apropiado para celebrar oficios religiosos; por lo que, a menudo, los curas visitan estos centros. En muchas escuelas privadas, preparan a los niños para que reciban el sacramento de la Comunión. Cabe mencionar, que se han creado instituciones educativas privadas que se rigen con un credo diferente al católico.

En algunos centros educativos, se celebran misas en varias ocasiones durante el año: inicio del curso lectivo, día de la madre, día del santo al que se dedicó la escuela y final de año, entre otras. Asimismo, los curas asisten a oír la confesión para esas mismas fechas y antes de Semana Santa.

\section{Relación escuela-Cuerpo de Bomberos}

Los grupos de las escuelas privadas también visitan el Cuerpo de Bomberos de la comunidad y si ahí no hay, coordinan con alguno para no perder la oportunidad de aprender de estos servidores públicos. No desean perderse las charlas que motivan, tanto a los docentes como a los estudiantes, a tener precaución para no sufrir abrasiones y a no causar quemas ni incendios más grandes que puedan dañar a otras personas y a las propiedades. Con estas explicaciones, los bomberos se ganan a los niños y estos los consideran sus amigos.

\section{Relación escuela-museos y parques}

Las visitas a los museos y a los parques zoológicos, ecológicos y arqueológicos son más numerosas que las realizadas por las escuelas públicas, pues tienen más facilidad de transporte y una mejor coordinación con padres o madres. En estos lugares puedan recibir una lección de Ciencias o de Estudios Sociales, con material auténtico. 


\section{Relación escuela-Ministerio de Salud}

En cuanto a la relación de estas escuelas con el Ministerio de Salud es muy limitada, pues, por lo general, cuando hay un brote de algunas enfermedades, los padres de estos niños pagan para que los vacunen. Pero sí aprovechan la experiencia de los funcionarios de este organismo y los invitan para llevar a cabo charlas o conferencias sobre hábitos de higiene, de salud y de alimentación.

\section{Relación escuela-INS}

Con el INS, las escuelas privadas mantienen la misma relación que las públicas, esto es como institución aseguradora de estudiantes. Posiblemente, en el sistema estatal de educación, no se aseguran tantos estudiantes como en el particular, pues aunque las cuotas no son muy altas, casi todos pueden pagar la prima de la póliza.

\section{Relación escuela-centros de salud privados}

Otro nexo que tienen las instituciones privadas es con los hospitales o clínicas privados, pues establecen convenios y así cuentan con algún centro de salud a mano y de inmediato, en el caso de alguna emergencia por parte de los estudiantes (caídas, golpes, peleas, desmayos, quebraduras y otros).

Las instituciones de educación privadas no se miden en cuanto a la seguridad de sus estudiantes; ellas saben que las emergencias no se van a dar todo el tiempo, ni que van a tener que desembolsar tanto dinero y, por otro lado, los padres y madres de familia, es probable, que se sientan contentos con esa preocupación por sus hijos.

\section{Relación escuela-empresa privada}

Muchos de los y las estudiantes de escuelas privadas son hijos de dueños de empresas, negocios e industrias, por lo que las autoridades de esas instituciones tratan de hacer que los niños y las niñas se acerquen a la empresa privada y que vayan conociendo, por mencionar un campo, lo que es la industria y cuáles son los pasos por seguir para elaborar un producto. Así, hacen visitas a diferentes empresas, como por ejemplo: Pozuelo, Jack's, Coca Cola, Pepsi y otras. Es notorio el entusiasmo de los y las estudiantes con estas visitas. 


\section{Relación escuela-Aviación Civil}

Algunos centros educativos privados tienen conexión con Aviación Civil, dado que muchas de las familias están acostumbradas a viajar. Así, las maestras solicitan una foto a los estudiantes y les elaboran un "pasaporte" y el día del "viaje" van al Aeropuerto Tobías Bolaños y los empleados imitan las actividades previas a emprender el vuelo, como son el revisar el equipaje (todos llevan un maletín) y sellarles su "pasaporte". Después, les permiten entrar en un avión y sentarse un rato.

Además de que los preparan para realizar trámites en un aeropuerto y ver los viajes como algo muy corriente en ellos, la visita les sirve para ilustrar aquellos medios de transporte más difíciles de usar como un helicóptero.

\section{Relación escuela-otras instituciones educativas}

Para terminar ${ }^{6}$, las instituciones de educación privadas se conectan, a menudo, con otras del mismo sistema, para lo cual existe una Asociación de Instituciones Educativas Privadas. La relación que llevan a cabo es de intercambio y, en ciertos casos, de coordinación de determinadas actividades.

En algunas ocasiones, esos intercambios no se limitan, exclusivamente, a escuelas nacionales, sino que también se llevan a cabo con escuelas internacionales, pues como apuntan García Hoz y Medina Rubio (1987), tal vez estén alejadas geográficamente, pero en algunos aspectos presentan coincidencias entre sí. Esas instituciones reciben a estudiantes costarricenses durante el mes de enero, en vista de que el calendario académico es diferente al costarricense, y no tienen problemas de pérdida de lecciones.

\section{Consideraciones finales}

Como se ha visto, la comunidad está conformada por una serie de instituciones de diferente tipo, las cuales mantienen entre sí una estrecha y provechosa relación. Esas relaciones se establecen para beneficio (directo o indirecto) de todos.

La escuela, como parte de la comunidad, debe buscar, constantemente, relaciones con otras instituciones, con el fin de utilizar el producto de

$6 \mathrm{Al}$ igual que con las escuelas públicas, esta rclación no agota las posibilidades; puede haber otras más. 
esa conexión para el beneficio de sus estudiantes, de ella misma y de la comunidad nacional. Ciertas relaciones que se llevan a cabo son más cercanas, más fijas y duraderas que otras, pues se realizan con instituciones que se encuentran en el mismo vecindario (instituciones públicas o gubernamentales, la mayoría de las veces) y, por lo general, hasta se conoce a los miembros de esas entidades. Otros nexos son más esporádicos, ya que las instituciones se encuentran alejadas y es difícil hacer el contacto.

Según los encuestados, algunas de esas relaciones se realizan más fácilmente porque se dan con organizaciones que tienen programas de extensión; es fácil establecer el nexo (si se sigue el trámite correspondiente) y no existen pagos adicionales, para ser recibidos; son instituciones que están muy anuentes a colaborar con la educación.

Una de las razones que dan los directores encuestados para obtener esas relaciones es que los estudiantes aprenden muchas cosas en el salón de clase, pero todo lo que aprenden, generalmente, es muy teórico, y estas actividades dan la posibilidad de llevarlas a la práctica.

Se debe tener presente, entonces, que los conocimientos se asimilan más fácilmente y permanecen por más tiempo (cuando no para siempre) si se aprende haciendo, con material auténtico y en un ambiente más amplio y en libertad. Todo esto es lo que se persigue cuando se establecen relaciones entre la escuela y otras instituciones.

Los nexos que se están planteando en este informe ya se han llevado a cabo en las instituciones educativas de las que son directores las personas encuestadas. Todas esas conexiones son posibles si se da una buena coordinación entre los sujetos participantes en el proceso educativo, esto es, entre las autoridades administrativas, los docentes, los padres y madres de familia, los y las estudiantes y, por supuesto, los que dirigen las instituciones con las que se establecerá la relación.

Como se notó en los datos presentados en el informe, la mayoría de las instancias que se relacionan con la escuela lo hacen tanto con centros educativos particulares como estatales; aunque se han planteado algunas conexiones diferentes realizadas por las escuelas privadas, casi todas ellas son comunes a los dos sistemas. Esto significa que ninguna de las modalidades educativas está en ventaja o en desventaja, sino que es fundamental el papel del docente y de la dirección en este tipo de actividades.

Curiosamente, tres posibles conexiones planteadas en la encuesta no fueron escogidas por ningún director, estas son: Bibliotecas, Correo y Teatro Nacional. 
Si se analizan los tipos de conexión planteados en este ensayo, se verá que la mayoría de las relaciones con otros entes es posible. Sin embargo, hay que reconocer que, en algunas ocasiones, no sólo se necesita la voluntad para realizar un convenio, un intercambio o una simple relación entre instituciones, sino que también se requiere de dinero para llevarlo a cabo. La falta de fondos para establecer alguna conexión no debería ser obstáculo para realizar otras.

Ha sido interesante, a pesar de las limitaciones de material, ahondar en este tema para tener un concepto de la realidad vivida en la educación costarricense. Ha sido enriquecedor conocer cómo cada institución se preocupa por ampliar los temas vistos en clase, para que sus estudiantes puedan darse cuenta de los aspectos que suceden fuera de las cuatro paredes los aspectos de la escuela y los tomen en cuenta a la hora de insertarse en las actividades de su entorno.

La información aportada por los directores encuestados resultó ser el insumo más importante para completar la investigación; sin su ayuda no se habría podido llevar a cabo. Después de haber completado la investigación, surge una inquietud: ¿qué piensan, sienten y experimentan los y las estudiantes cuando se realizan las actividades con las otras instituciones? Sería interesante investigar acerca de este tema. ¿Quién se atreve a realizar ese trabajo? 


\section{Referencias}

Cárdenas, M. (1990). Antología para el curso Escuela y comunidad. (1 $1^{\mathrm{a}}$ Reimpres). San José, Costa Rica: EUNED.

Cassany, D. (1999). Desde lo cotidiano. Disponible en: http://www.cep.edu.uy/ RedDeEnlace/TizayPizarron/RevMtros199b.htm

García, V. y Medina, R. (1987). Organización y gobierno de centros educativos. Madrid: EDITORIAL RIALP.

Romero, R. (s.f.). La educación y la escuela en el banquillo. Opiniones y notas de FLATEC (Federación Latinoamericana de Trabajadores de la Educación y la Cultura). Disponible en: http://www.flatec.org/notas/opiniones/ opinión_6htm

Relaciones entre la escuela y otras instituciones de la comunidad (Feb. 2004). Encuesta realizada a diez directores de escuelas públicas y privadas. 